\title{
Characterization of Differences in Functional Connectivity Associated with Close-Range Blast Exposure
}

\author{
Meghan E. Robinson, ${ }^{1-3}$ Dustin C. Clark, William P. Milberg, ${ }^{2,4,5}$ \\ Regina E. McGlinchey, ${ }^{2,4,5}$ and David H. Salat ${ }^{1,2,6}$
}

\begin{abstract}
Despite the prevalence of blast injuries in recent overseas conflicts, knowledge of their impact on neural health is lacking. We have recently published work demonstrating differences in functional magnetic resonance imaging (fMRI) connectivity that were specific to close-range blast exposure (CBE), as opposed to other prevalent military-related factors. Here, we replicate this finding in an independent sample of 135 veterans, again finding that CBE, regardless of concussion, is predictive of persistent changes in brain physiology. Although there was weak overlap anatomically, in both samples, the group differences could be described as spreading of anticorrelation. Using the combined sample, we now seek to identify likely mechanisms that could bring about this effect. We compared participants with $(n=116)$ and without ( $n=153$ ) CBE by analyzing two networks through group difference maps and correlation distributions to assess spatially homogenous and heterogeneous effects. As boundaries between positive and negative correlations in fcMRI are determined by noise covariates, we compared analyses with and without global signal regression. We found evidence of widespread altered connectivity that was spatially heterogeneous across participants, and that the role of global signal regression was network dependent. These findings are not consistent with expected results from damaged white matter or impaired neural function. Rather, potential biological interpretations include disrupted cerebral blood flow or impaired neurovascular coupling, which have each been observed in animal models of blast exposure. Further targeted work will be necessary to distinguish the contribution of each of these mechanisms to producing changes in brain function associated with CBE.
\end{abstract}

Keywords: adult brain injury; military injury; MRI

\section{Introduction}

$\mathbf{C}$ OMBAT-RELATED BLAST EXPOSURES are common in service members who served in Afghanistan and Iraq; over 50\% of soldiers returning from Iraq reported being near two or more improvised explosive devices (IEDs), in many cases being exposed to high energy explosives, now survivable because of advanced vehicle and body armor. ${ }^{1}$ Hence, the prevalence of survivable blastrelated injuries is unprecedented; therefore, it is not surprising that there are critical gaps in knowledge regarding the neurobiological consequences of exposure to these blasts. Animal and human research suggests that blast exposure is associated with a number of pathological changes in brain tissue. In animal models, primary blast exposure, without accompanying blunt trauma, is associated with altered gene expression, ${ }^{2}$ brain chemistry, ${ }^{3}$ and neuroimaging findings. ${ }^{4}$ Neuroimaging work in humans ${ }^{5-7}$ has demonstrated that exposure to blast, even without diagnostic symptoms of concussion, results in measurable changes in the brain, and may even alter brain aging trajectories. ${ }^{8}$

Our recent work ${ }^{6}$ revealed differences in functional connectivity magnetic resonance imaging (fcMRI) in veterans of Operations Enduring Freedom, Iraqi Freedom, and New Dawn (OEF/OIF/ OND) reporting exposure to blast, regardless of whether the blast was associated with concussion. Specifically, we found that in the group reporting close-range blast exposures (CBE) (self-reported to be within $10 \mathrm{~m}$ ), areas of anticorrelation (i.e., negative correlation) to the default mode network (DMN) extended into the somatomotor cortex, unlike the no-CBE group. Critically, changes in fcMRI were specific to CBE, and were not dependent on associated concussion symptoms; that is: fcMRI alterations for veterans with CBE were

\footnotetext{
${ }^{1}$ Neuroimaging Research for Veterans (NeRVe) Center, ${ }^{2}$ Translational Research Center for TBI and Stress Disorders (TRACTS) National Research Center, and ${ }^{5}$ Geriatric Research, Education, and Clinical Center (GRECC), VA Boston Healthcare System, Boston, Massachusetts.

${ }^{3}$ Department of Neurology, Boston University School of Medicine, Boston, Massachusetts.

${ }^{4}$ Department of Psychiatry, Harvard Medical School, Boston, Massachusetts.

${ }^{6}$ A. A. Martinos Center for Biomedical Imaging, Charlestown, Massachusetts.
} 
equal for those with and without concussion symptoms. Further, changes in fcMRI were not attributable to factors such as posttraumatic stress disorder (PTSD), sleep quality, pain, or length of or time since deployment. Finally, we observed these changes months to years after the exposures, suggesting that blast-related alterations in fcMRI are long lasting, if not permanent. We observed no similar effect from blasts at greater distances (i.e., $25-100 \mathrm{~m}$ ), or as a result of concussion from blunt trauma. We concluded that blast exposures at close range - regardless of concussion status - play a unique and largely unexplored role in the brain health of veterans.

The goal of this study is to identify likely biological mechanisms that may have brought about these previously observed changes in functional connectivity in veterans with CBE. Because fcMRI is an indirect measure of neural function, ${ }^{9,10}$ and, therefore, is influenced by nonspecific changes in underlying biological processes including neural function, ${ }^{11}$ we seek to further explore the information contained in the fcMRI measurement to attempt to narrow the potential biological interpretations of the effects associated with CBE. fcMRI is often conceptualized as arising from brain regions that co-activate through white matter connections. ${ }^{12}$ Glial cells in the neurovascular unit then mediate a complex metabolically driven vascular response to local activations, resulting in changes in magnetic properties that are MR-visible. ${ }^{13}$ In healthy brains, fcMRI is largely a reliable tool for the study of brain network activity, and as expected, pathological changes such as degenerating white matter pathways or altered neural function result in aberrant fcMRI patterns. ${ }^{14}$ In addition to these primarily neutrally driven deviations, non-neural sources of variation, which are assumed stable in healthy populations, may affect the fcMRI signal in pathological conditions. For example, pathological changes in the glial neurovascular unit may result in neural activity (that is largely preserved) to be poorly translated into a vascular response, ${ }^{15}$ or the vasculature may be unable to comply with metabolic demands of neurons. ${ }^{16,17} \mathrm{It}$ is currently unclear which, if any, of these mechanisms may explain the observed differences in fcMRI in individuals with CBE, as each is corroborated through animal studies of blast (see Table 1$)^{18-29}$.

In general, fcMRI is used to estimate coordinated neural activity, by comparing correlation values either across regions or across participants. However, the technique is susceptible to shared noise in the signal across the brain, such as that arising from physiological variation. ${ }^{30}$ This noise can bias the correlation measurements;

Table 1. Summary of the Effects of Blast Overpressure on Biological Tissues Based on Animal Model Studies

\begin{tabular}{|c|c|}
\hline White matter & $\begin{array}{l}\text { Diffuse axonal injury, }{ }^{18} \text { disruption of } \\
\text { axonal microtubles, }{ }^{19} \text { apoptosis of } \\
\text { oligodendrocytes }{ }^{20,21}\end{array}$ \\
\hline $\begin{array}{l}\text { Gray matter/ } \\
\text { Neural function }\end{array}$ & $\begin{array}{l}\text { Neuronal loss, }{ }^{22} \text { altered } \\
\text { electrophysiological response, }{ }^{21} \text { gene } \\
\text { expression, }{ }^{21} \text { abnormal tau } \\
\text { expression, }{ }^{3,23} \text { oxidative stress }{ }^{24,25}\end{array}$ \\
\hline $\begin{array}{l}\text { Neurovascular } \\
\text { coupling (glial) }\end{array}$ & $\begin{array}{l}\text { Collapsed capillaries with hypertrophic } \\
\text { astroglial endfeet, }{ }^{19} \text { subcortical } \\
\text { gliosis, }{ }^{20,21} \text { astrocytic and microglial } \\
\text { activation, }{ }^{25,26} \text { inflammation, }{ }^{26,27} \text { blood- } \\
\text { brain barrier permeability }{ }^{21,25}\end{array}$ \\
\hline Vascular integrity & $\begin{array}{l}\text { Molecular evidence of vascular } \\
\text { pathology, }{ }^{28} \text { cerebral vasospasm, } \\
\text { altered cerebral blood flow, } \\
\text { microvasculature changes }{ }^{21}\end{array}$ \\
\hline
\end{tabular}

therefore, efforts are made in post-processing to limit its influence. One method for achieving this is to regress out time-dependent activity common to the entire brain, using what is known as global signal regression (GSR). Somewhat similarly to subtracting the mean correlation, GSR divides the brain into networks that are half positive and half anticorrelated, similar to what is seen during tasks. $^{31}$ A problem arises, however, because with GSR, anticorrelation would arise mathematically regardless of its biological appropriateness, ${ }^{32}$ and without GSR, the brain is almost entirely positively correlated. ${ }^{33}$ Although there is evidence that in healthy individuals GSR provides a reasonable estimation of a neurophysiologically meaningful reference point, ${ }^{34}$ the accuracy of that estimate may depend on non-neural factors such as vascular function, which could vary between groups. ${ }^{35}$ This could be why the global signal itself can hold clinically meaningful information. ${ }^{36,37}$

The major finding of our previous work was a spread of the anticorrelation to a DMN seed into the somatomotor cortex. The role of the somatomotor cortex was unclear, as the global signal will determine the "zero-point" between positive and negative correlations, suggesting a change in the signal driving the brainwide average. Therefore, in the present study, we examined the DMN, the global waveform, and the dorsal attention network (DAN), an anticorrelated network. ${ }^{31}$ We calculated group difference maps, which assume spatial homogeneity; as well as distributions of correlations, which do not. Each analysis was conducted for each network with and without GSR. The results provide further characterization of the nature of the differences between veterans with and those without CBE, and offer directions for the study of neurophysiological mechanisms underlying blast neurotrauma.

\section{Methods}

\section{Participants}

Participants, image acquisition, and behavioral covariates have been described in detail elsewhere. ${ }^{6,38}$ Briefly, individuals in this report were the first 400 participants in the Translational Research Center for TBI and Stress Disorders (TRACTS), a VA Rehabilitation Research and Development National Research Center. Although individuals with major medical and mental health issues not addressed by the study were excluded $(n=21)$, the cohort is notable for prevalent psychiatric conditions including PTSD, chronic pain, sleep disorder, and depression at rates that are representative of this veteran population (see Table 2). For this study, participants were also not included if their MRI data were not acquired or were of poor quality $(n=76)$, or if they had not completed all measures that were used as covariates $(n=34)$. This resulted in 269 participants included in the analysis, 134 of which have been reported in a 2015 study by Robinson and coworkers. ${ }^{6}$ Blast history was acquired through the Boston Assessment of TBILifetime (BAT-L) semi-structured interview. ${ }^{39}$

\section{Image acquisition}

Resting state fMRI data (repetition time [TR]/echo time [TE], $3000 / 30 \mathrm{~ms}$; flip angle, $90 ; 3 \times 3 \times 3.75 \mathrm{~mm} ; 38$ slices) were collected on a 3T Siemens TIM Trio for two 6 min runs during which participants were asked to keep their eyes open and stay awake. An anatomical scan was collected in the same session for registration.

\section{Image analysis}

Neuroimaging data were analyzed using tools from FreeSurfer, ${ }^{40-42}$ AFNI, ${ }^{43}$ FSL, ${ }^{44}$ and MATLAB (MathWorks; Natick, MA). For all subject-level general linear model (GLM) analyses unless otherwise noted, age, gender, IQ, and score on the Clinician 
Table 2. Demographics for Close-Range Blast Exposure (CBE) and No CBE on Deployment-Related Factors

\begin{tabular}{|c|c|c|c|c|}
\hline & Total $(\mathrm{n}=287)$ & $C B E(\mathrm{n}=128)$ & No $C B E(\mathrm{n}=159)$ & $\mathrm{p}$ value \\
\hline Age & $31.9 \pm 8.3$ & $31.7 \pm 8.3$ & $32.1 \pm 8.3$ & 0.678 \\
\hline Men & $89.6 \%$ & $93.8 \%$ & $86.2 \%$ & $\mathbf{0 . 0 3 7}$ \\
\hline Education & $13.9 \pm 1.9$ & $13.8 \pm 1.9$ & $14.0 \pm 1.8$ & 0.315 \\
\hline WTAR estimated IQ & $102.5 \pm 11.8$ & $102.0 \pm 12.2$ & $102.9 \pm 11.5$ & 0.489 \\
\hline $\begin{array}{l}\text { OEF/OIF deployments } \\
\text { Number of deployments } \\
\text { Months since deployment } \\
\text { Duration (months) } \\
\text { Combat Exposure }\end{array}$ & $\begin{array}{c}1.4 \pm 0.8 \\
40.1 \pm 31.9 \\
13.8 \pm 9.0 \\
16.5 \pm 11.6\end{array}$ & $\begin{array}{c}1.6 \pm 0.8 \\
46.6 \pm 33.6 \\
16.0 \pm 9.4 \\
23.5 \pm 10.3\end{array}$ & $\begin{array}{c}1.2 \pm 0.8 \\
34.6 \pm 29.4 \\
12.0 \pm 8.2 \\
10.7 \pm 9.2\end{array}$ & $\begin{array}{r}<0.001 \\
0.002 \\
<0.001 \\
<0.001\end{array}$ \\
\hline $\begin{array}{l}\text { Traumatic brain injury (TBI) } \\
\text { Number of blasts } \\
\text { Number of lifetime TBIs } \\
\text { Number of military TBIs } \\
\text { Loss-of-consciousness (min) } \\
\text { Post-traumatic amnesia (min) } \\
\text { Altered mental state (min) }\end{array}$ & $\begin{array}{c}4,1,14 \\
1,0,4 \\
0,0,1 \\
0,0,1 \\
0.2,5,5 \\
5,0,55\end{array}$ & $\begin{array}{c}11.5,5,28 \\
1.5,1,3 \\
1,0,2 \\
0.1,0,1.9 \\
1,0,8 \\
15,0.9,106\end{array}$ & $\begin{array}{c}1,0,4 \\
1,0,2 \\
0,0,0 \\
0,0,1 \\
0,0,5 \\
1.2,0,19.9\end{array}$ & $\begin{array}{r}\mathbf{0 . 0 0 8} \\
<\mathbf{0 . 0 0 1} \\
\mathbf{< 0 . 0 0 1} \\
0.339 \\
0.229 \\
0.080\end{array}$ \\
\hline $\begin{array}{l}\text { Psychiatric conditions } \\
\text { Post-traumatic stress disorder } \\
\text { Mood disorders } \\
\text { Panic disorder } \\
\text { Social phobia } \\
\text { Generalized anxiety disorder } \\
\text { Other anxiety disorder } \\
\text { Alcohol abuse/ dependence } \\
\text { Other substance abuse/ dependence } \\
\text { Current pain } \\
\text { Average pain in } 30 \text { days } \\
\text { Sleep impairment } \\
\text { Sleep quality }\end{array}$ & $\begin{array}{c}59.1 \% \\
22.7 \% \\
4.9 \% \\
5.9 \% \\
6.6 \% \\
4.6 \% \\
12.6 \% \\
3.5 \% \\
68.4 \% \\
28.9 \pm 24.8 \\
76.2 \% \\
9.6 \pm 4.8\end{array}$ & $\begin{array}{c}77.3 \% \\
27.3 \% \\
4.7 \% \\
4.7 \% \\
7.1 \% \\
5.5 \% \\
11.0 \% \\
3.9 \% \\
79.3 \% \\
35.7 \pm 26.6 \\
86.9 \% \\
11.0 \pm 4.6\end{array}$ & $\begin{array}{c}44.3 \% \\
18.9 \% \\
5.0 \% \\
6.9 \% \\
6.3 \% \\
3.8 \% \\
13.8 \% \\
3.1 \% \\
60.0 \% \\
23.5 \pm 22.0 \\
67.6 \% \\
8.5 \pm 4.6\end{array}$ & $\begin{array}{r}<\mathbf{0 . 0 0 1} \\
0.088 \\
0.905 \\
0.436 \\
0.788 \\
0.483 \\
0.476 \\
0.727 \\
<\mathbf{0 . 0 0 1} \\
<\mathbf{0 . 0 0 1} \\
<\mathbf{0 . 0 0 1} \\
<\mathbf{0 . 0 0 1}\end{array}$ \\
\hline
\end{tabular}

${ }^{\mathrm{a} B e c a u s e}$ of skewed distribution, the median and interquartile range are reported, rather than mean and standard deviation. Bold indicates significant differences at $p<0.05$.

WTAR, Wechsler Test of Adult Reading; OEF, Operation Enduring Freedom; OIF, Operation Iraqi Freedom.

Administered PTSD scale ${ }^{45}$ were included as nuisance regressors. Multiple comparisons correction was completed using FreeSurfer's mri_glmfit-sim with vertex- and clusterwise thresholds of 0.05 .

Replication. Of the available 269 participants, those that were not included in the 2015 study by Robinson and coworkers ${ }^{6}$ $(n=135)$ were analyzed in the same manner as that study to test the reliability of the findings. Preprocessing included sampling to and smoothing on the cortical surface, motion correction, band pass filtering $(0.01-0.10 \mathrm{~Hz})$, and regression of motion traces and average time course from white matter, ventricles, and global mean. Resting state fMRI scans for participants with $(n=116)$ and without $(n=153)$ self-reported CBE, regardless of concussion status, were compared on connectivity to a seed region in the superior third of the isthmus of the cingulate, as defined by each participant's nativespace anatomic parcellation by FreeSurfer. ${ }^{46}$

Global signal regression. For the full sample $(n=269)$, the analysis described was repeated without using the GSR. Then, the global waveform itself was used as a seed time course using the methods described producing a vertex-wise correlation for comparison across groups.

Task positive network. To assess the role of so-called "taskpositive" networks, which are anticorrelated to the DMN, the inferior half of the postcentral sulcus ${ }^{46}$ was used as a seed for the
DAN, using the same preprocessing and statistical analysis as that described.

Correlation distributions. Correlation value distributions over the cortex were generated for each participant for the surfaces described. These distributions do not contain spatial information, but rather describe how often vertices with certain correlation values occur. These were then averaged within each group, and $t$ tests for each bin were used to determine significant differences.

\section{Results}

\section{Demographics}

The groups did not differ in mean ( $t$ test, $p>0.8$ ) or distribution (Kolmogorov-Smirnoff, $p>0.9$ ) of volume-to-volume motion or number of censored volumes. They did, however, differ on a number of other military-related factors (Table 2).

\section{Replication of previous finding}

The main findings of our previous work were replicated. We found differences between veterans with and without CBE that could be described as spreading of anticorrelation, although the regions that met significance were more ventral, and stronger on the left rather than the right (Figs. 1 and 2). Somatomotor cortex was minimally significant in the replication. Secondary analyses similar 


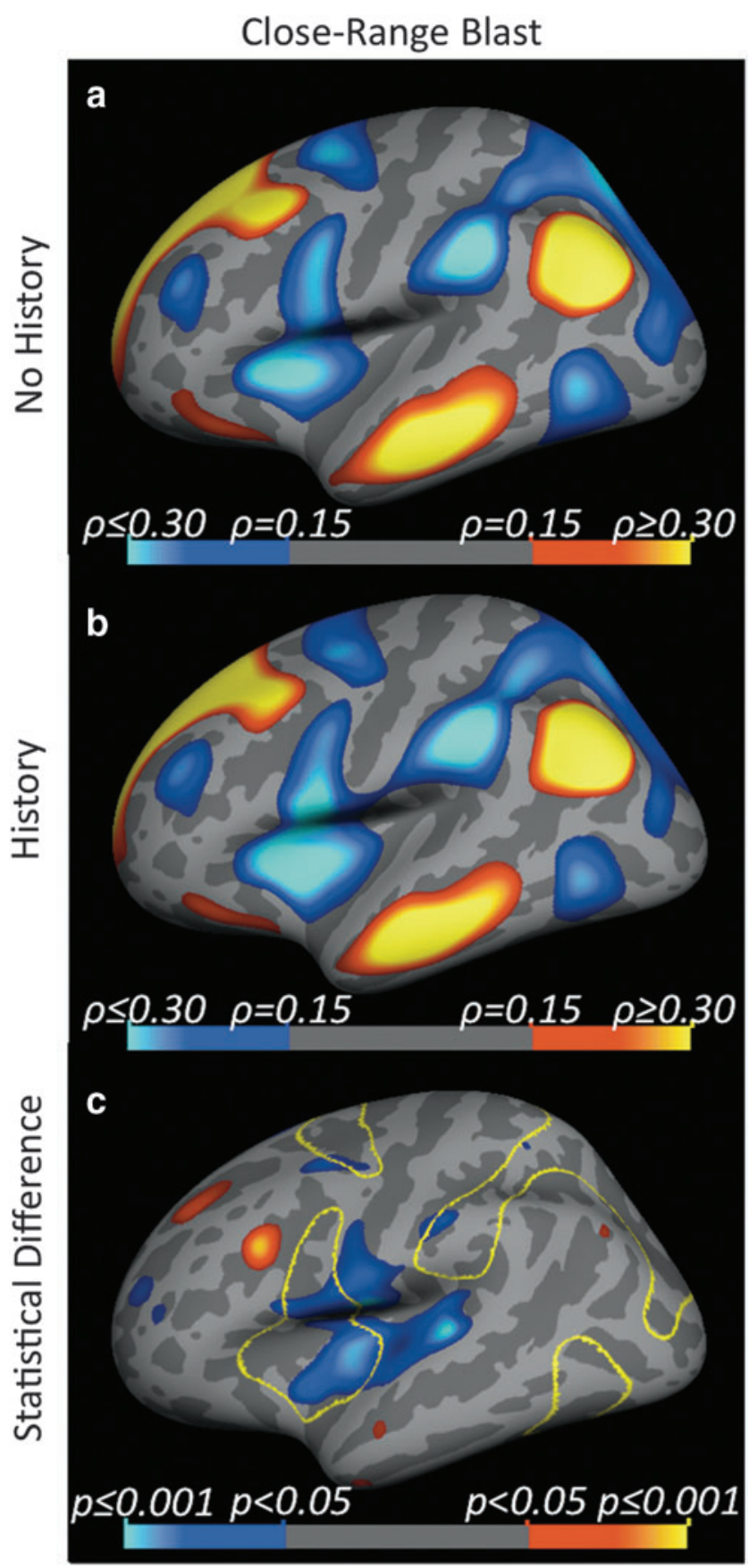

FIG. 1. Replication of group differences in close-range blast exposure (CBE). For 135 participants, none of whom were included in the prior work, groups were determined based on selfreport of being within $10 \mathrm{~m}$ of a blast. Groups were compared on connectivity to a seed region in the superior third of the isthmus of the cingulate, a hub in the default mode network. (a) Group mean for participants without CBE. (b) Group mean for participants with CBE. (c) Statistical difference between the two groups, displayed at uncorrected $p<0.05$. Clusters that survive correction for multiple comparisons are shown in Figure 2. Note that in both groups, the area of correlation to the seed (yellow-red in [a] and [b]) is similar; however, the area of anticorrelation (blue-cyan in [a] and [b]) covers a greater spatial extent in the CBE group. We refer to this effect as "spreading of the anticorrelation." to those in the study by Robinson and coworkers ${ }^{6}$ can be found in Table S1, demonstrating that these results are independent of other military-related conditions such as PTSD and military concussion (see online supplementary material at http://www.liebertpub.com).

\section{Role of global signal regression}

Figure 3 a shows the average of the global signal connectivity map for all participants with seeds and significance regions labeled. We found no group differences in global waveform seed connectivity (Fig. 3b) for either vertex-wise analyses or whole-brain averages, suggesting that there were no overt differences in global signal. Using all participants $(n=269)$, the DMN analysis was conducted with and without GSR (Fig. 4a,b). Without GSR, groups did not significantly differ. Whole-brain averages were included as subject-level covariates to replace the GSR, resulting in vertex-wise significance differences in similar regions to the spreading anticorrelation observed; however, these did not survive correction for multiple comparisons.

\section{Task positive network}

We found no significant difference between groups in DAN connectivity (Fig. 4c,d).

\section{Correlation distributions}

Similar to the group maps, with GSR, the DMN had group differences in distributions of cortical correlations, but the DAN did not (Fig. 5a,c). However, unlike the group maps, both networks demonstrated significant group differences without GSR (Fig. 5b,d), demonstrating that GSR processing does not create the group difference. Connectivity to the global waveform differed on two out of 50 histogram bins, which is the rate expected by chance. Vertices with high correlation to the DMN seed (without GSR) are shown in Figure 6 as an overlap map. It is of note that within the CBE group, regions that are highly correlated to the DMN seed are more widespread, although core areas of the DMN are quite similar.

\section{Discussion}

In this report, we have, first, replicated our previous finding of connectivity differences associated with $\mathrm{CBE}$ in an independent sample of $135 \mathrm{OEF} / \mathrm{OIF} / \mathrm{OND}$ veterans. Confirmation of this result is important, as it establishes the utility of proximity cutoffs as a meaningful indicator of blasts that may lead to brain injury. The replication was not exact; the anatomical overlap between the two samples was notably poor. However, in both cases, the effect can be described as a spread of the anticorrelation (Fig 1). This may suggest that this type of effect, spreading of anticorrelation, is not highly spatially constrained and may be a more general phenomenon of the global physiology. The remainder of the report seeks to identify potential biological mechanisms that could promote a spread in anticorrelation by simultaneously considering multiple aspects of the functional connectivity data. We believe that this is indicative of disturbance in either vascular health or neurovascular coupling, and our reasoning is as follows.

We examined two networks: the DMN, which was used in the original finding and the replication; and the DAN, one of the networks consistently anticorrelated to the DMN. ${ }^{31}$ Each network was analyzed both with and without the use of GSR. Group difference maps were calculated along with the distribution of vertex-wise correlations across the cortex. We found that the DMN was associated with spreading anticorrelation when GSR was used, but not without GSR. For the DAN, no significant differences in the group 


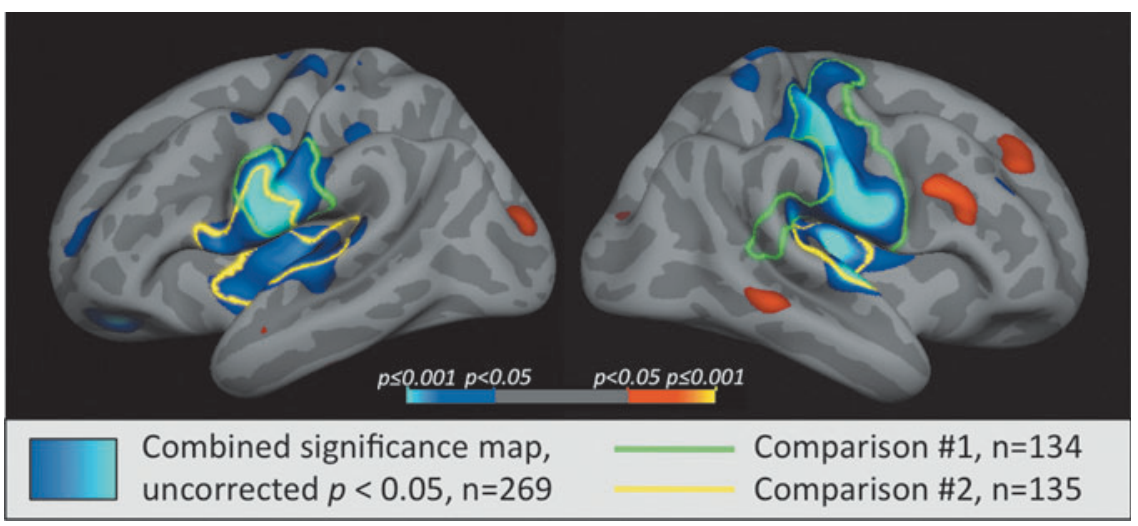

FIG. 2. Overlap of significance maps across replication groups. Outlines of significance regions from the original finding ${ }^{6}(n=134)$ and the replication $(n=135)$ are shown in outlines over the significance map of the combined group. Although in all cases the effect can be described as a "spreading of the anticorrelation," the anatomical overlap between replication studies is poor, especially in the right hemisphere.

maps were seen with or without GSR (Fig. 4). Both networks had significant differences in the correlation distribution when GSR was not used, but only the DMN differed when it was (Fig. 5). Finally, we found no significant differences in the group map of connectivity to the global signal.

Considering only the results for the DMN, these results seem to indicate DMN hyperconnectivity to regions that are heterogeneous across participants. These positive connections would then bias the global waveform to be similar to the DMN time course, resulting in more negative connections relative to it. Although this is corroborated by the overlap map of DMN connections (Fig. 6), and similar results have been reported elsewhere in association with nonconcussive head trauma, ${ }^{47}$ it does not explain the results we observed for the DAN. In the DAN, distributions of correlations prior to GSR were even more strikingly different between groups. However, after GSR, the distributions were not different, and the group map showed no significant differences, unlike in the DMN. To explain these results, we examined the topology of the global signal connectivity itself. We found that regions of high correlation to the global signal were very similar to the significance regions, and that hubs of the DMN network were also areas of low global correlation. In this framework, we saw that areas of the brain that became significant after GSR were those most similar to the global signal, and, therefore, if CBE resulted in less correlation to the global signal, there was more "room" to move toward the DMN than toward the DAN. Thus, we find an explanation consistent with all of our findings; namely, that with CBE, areas of the brain become less related to the global signal and begin to appear more
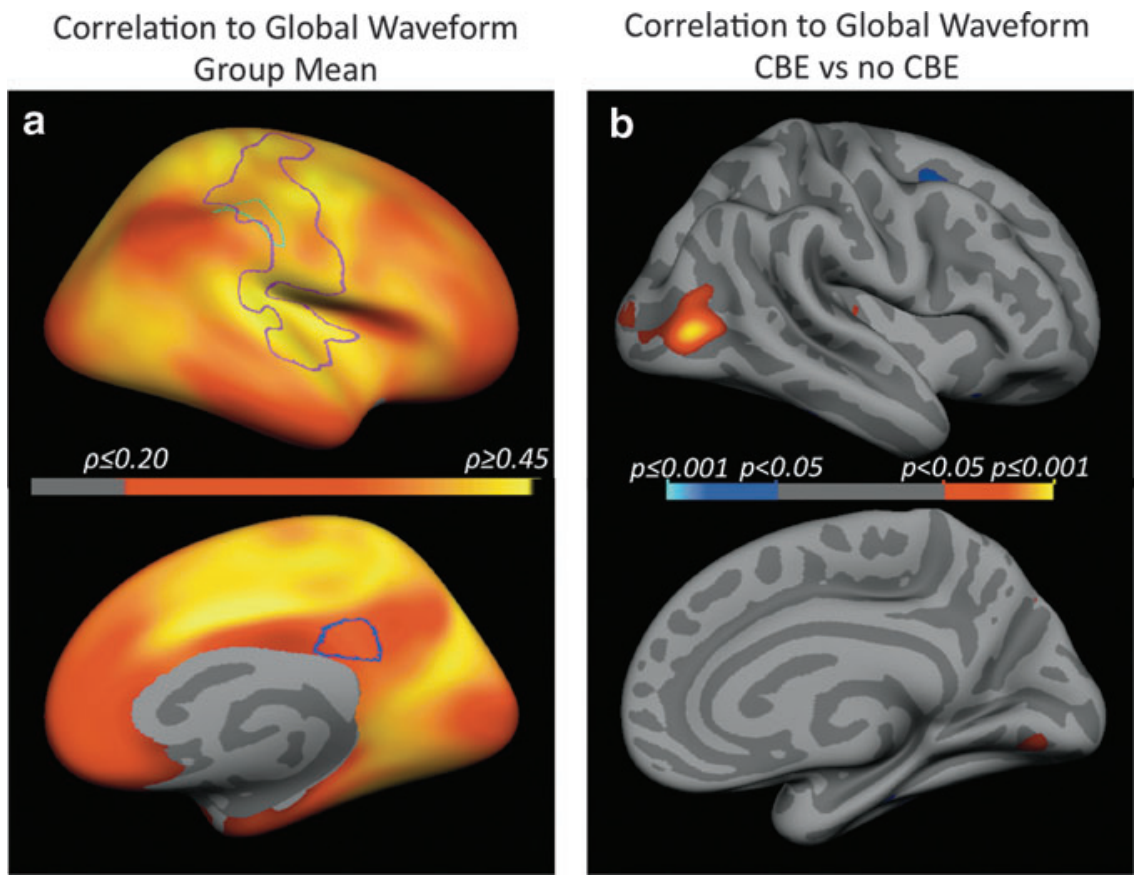

FIG. 3. Global Signal. Panel (a) shows the mean correlation to the global signal averaged over all participants. Seed regions for each network are labeled, as well as the significance region identified in the main analysis. Purple, significance region; green, dorsal attention network seed; blue, default mode network seed. Panel (b) shows the group difference map for correlation to the global signal. No clusters survived correction for multiple comparisons. 

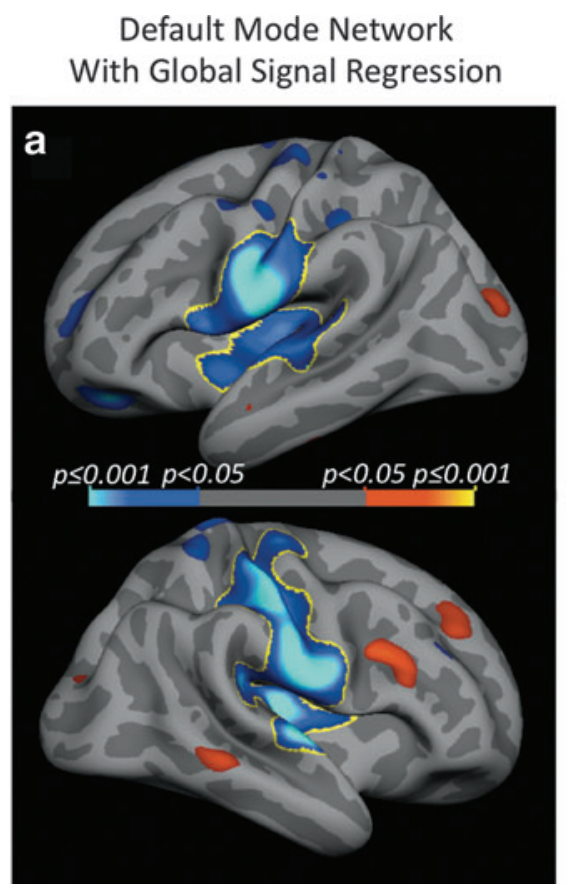

Dorsal Attention Network With Global Signal Regression

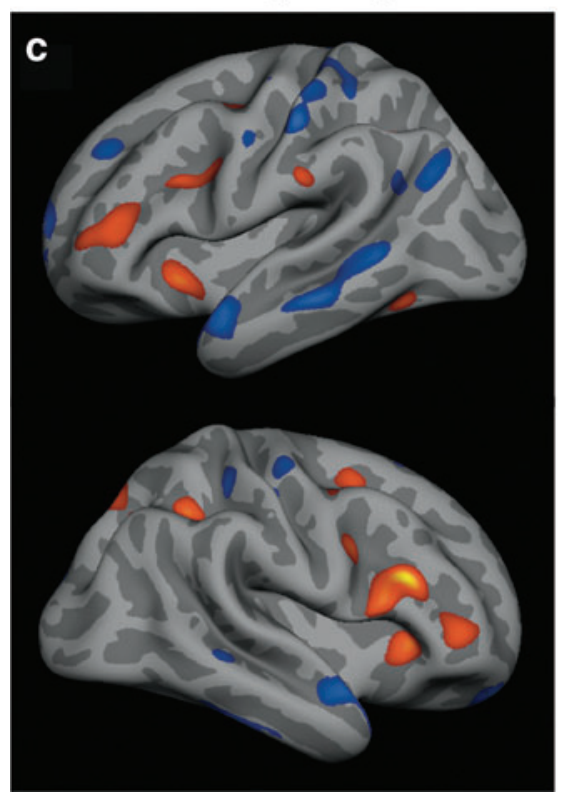

Default Mode Network without Global Signal Regression

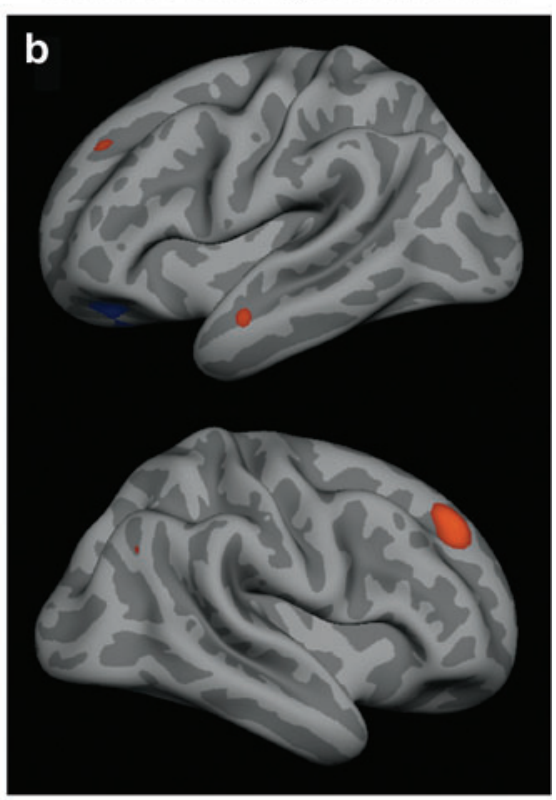

Dorsal Attention Network without Global Signal Regression

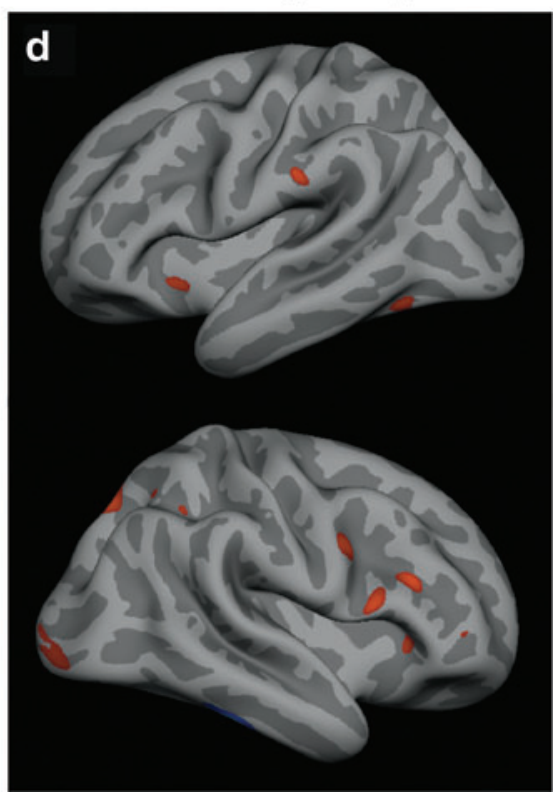

FIG. 4. Comparison of correlation analyses. Group difference statistical maps are shown for four correlation analyses. (a) Main analysis: correlation to seed in the superior third of isthmus of the cingulate, a default mode network (DMN) hub, using the global signal regression. (b) Correlation to the DMN seed without using the global signal regression. (c) Correlation to the seed in the inferior half of the postcentral sulcus, a dorsal attention network (DAN) hub, using global signal regression. (d) Correlation to the DAN seed without using global signal regression. Note that only the first analysis includes clusters that survived correction for multiple comparisons (denoted with yellow outline).

DMN-like, although the specific regions in which this occurs are not consistent across individuals.

There are a number of biological mechanisms that may result in these phenomena. Drawing from previously identified tissue injuries associated with blast injuries (Table 1), with white matter damage or impaired neural function, we would expect that the spreading anticorrelation to the DMN would arise from less DMN input to the area (either because of neuronal loss in the DMN or impaired axonal transport from it) such that task-positive network activity dominates. As such, DMN connectivity with and without GSR would be similar, and we would have expected that increased anticorrelation to the DMN would have been associated with complementary findings in the DAN, which were not observed. Rather, altered cerebral blood flow and microvascular changes, or impaired neurovascular coupling were the more likely mechanisms, although we cannot exclude contributions from neural 


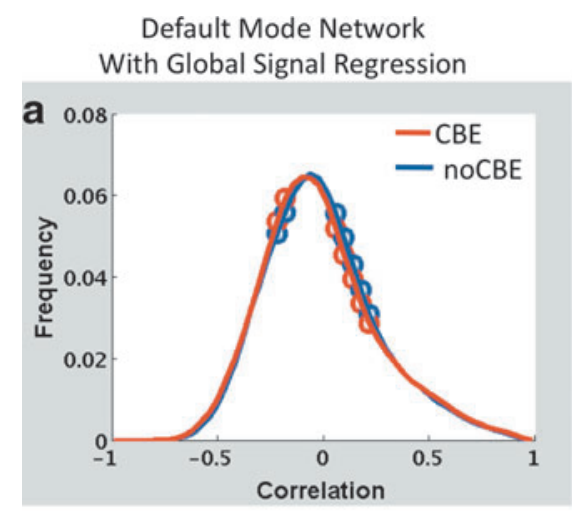

Dorsal Attention Network With Global Signal Regression

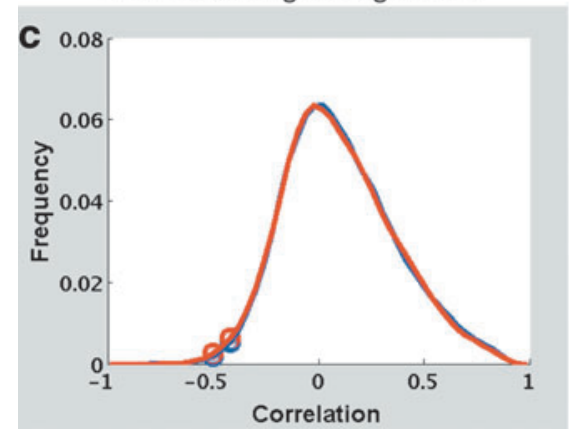

Default Mode Network without Global Signal Regression

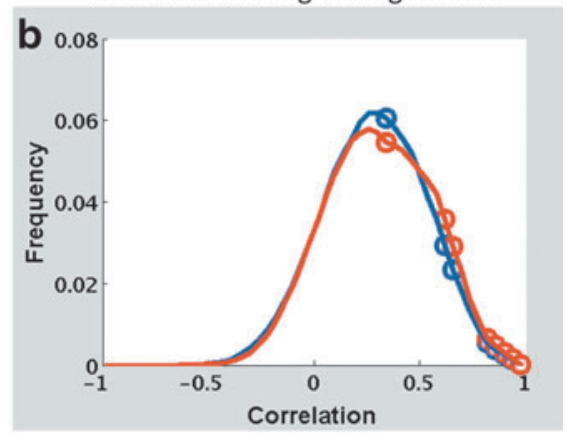

Dorsal Attention Network Without Global Signal Regression

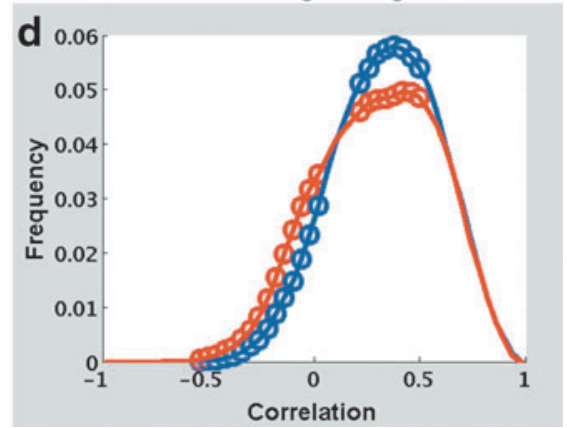

FIG. 5. Group differences in correlation distribution. Histograms of vertex-wise correlations across the cortex are shown. Mean histogram for participants with close-range blast exposure (CBE) is shown in red, and for those without CBE it is shown in blue. Significant differences $(p<0.05)$ at each bin are indicated with circles. Panels a-d show the same analyses as shown in Figure 4 . Note that there are significant differences between groups, independent of inclusion of global signal regression.

impairment through this study. Either microvascular changes or impaired neurovascular coupling could result in inconsistent translation of typical neural activity into blood-oxygen-level-dependent (BOLD) signal. Histopathological work in veterans exposed to blasts has shown evidence of widespread glial injury, ${ }^{48}$ consistent with impaired neuro-vascular coupling, as well as prevalent vascular issues. ${ }^{49}$ Spatial inhomogeneity across participants is not surprising given the nature of the injury, and this is a consistent theme throughout blast concussion literature. ${ }^{50-54}$ Ultimately, it is unlikely that exposure to detonated munitions affects only one aspect of the brain's functions, and it may be that some combination of effects is producing the results that we observed. Although this report is a step toward understanding the biological mechanisms of blast injury in the brain, there are still many issues that need to be addressed before a complete mechanistic understanding of blastrelated brain injury can be defined.

It has been reported previously that the use of the GSR in connectivity studies can produce regions of statistically significant differences, which do not necessarily reflect the underlying true differences in groups. ${ }^{55}$ The analyses presented here appear to form

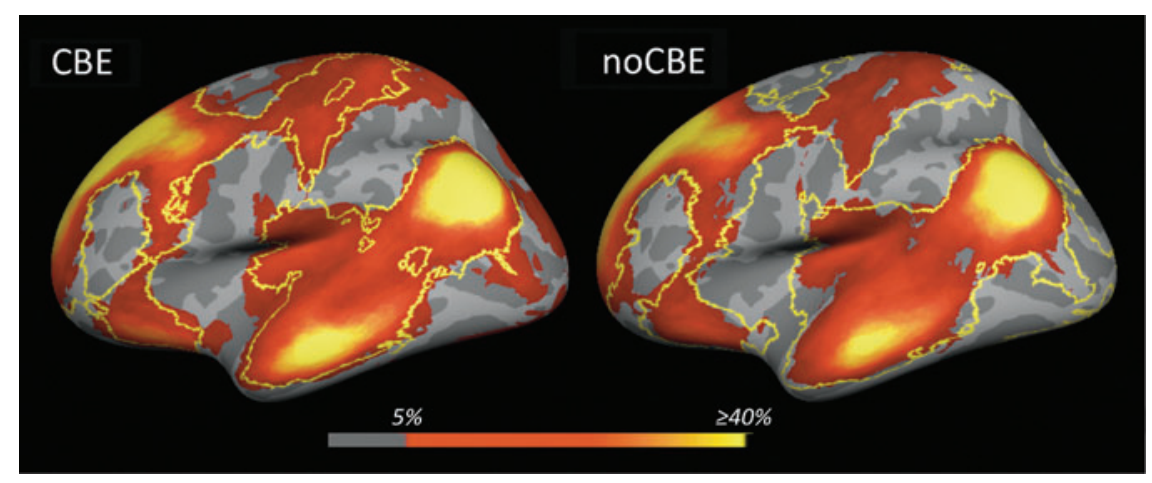

FIG. 6. Localization of vertices with high correlation to the DMN seed. Vertices with correlation values of 0.66 or greater to the default mode network (DMN) seed (see Figure 5b) were identified for each participant. Areas where this criteria was met are shown as overlap maps indicating the percent of participants in each group where this was the case. For reference, each map includes the outline of the other group's map. Areas of high correlation are more widespread in the close-range blast exposure (CBE) group, although no area outside the core DMN hubs represents a large percentage of the group. 
another example of this, where a group difference that was present even without GSR in the distribution of correlations was observed as spreading anticorrelation in the group maps using GSR. It is of note that without the use of the GSR, no differences in the DMN connectivity group maps were detected, even with twice as many participants as either analysis using GSR. Therefore, like others, ${ }^{37}$ we endorse the need for caution in interpreting results of connectivity analyses using this common technique, especially given the many neurophysiological processes that may differ between groups to ultimately produce a change in observed functional connectivity. However, we note that rather than creating spurious group differences driven by background noise, we were able to detect a group difference that was present in a different aspect of the BOLD activity.

This study adds to a growing understanding that blast exposures need not result in concussion symptoms at the time of the event to produce changes to the brain, and that proximity to blast may serve as a better estimate of injury severity than reported symptoms. Further work is needed to completely understand this phenomenon, including further in vivo studies and histopathological work that is specifically aimed at nearby blast exposure. Our first study ${ }^{6}$ demonstrated that, among the different ways of assessing head trauma, CBE is highly predictive of differences in functional connectivity. In this study, by using multiple methods of examining that functional connectivity, we found that the most likely biological interpretation of these fcMRI differences was either vascular or glial disturbance. These interpretations are consistent with our findings that nearly half of participants who have had CBE do not report overt symptoms at the time of exposure. Further research to extend these findings may include studies of cerebrovascular reactivity, hemodynamic coupling, or electrical recordings of neural function.

\section{Acknowledgments}

This research was supported by the Translational Research Center for TBI and Stress Disorders (TRACTS), a VA Rehabilitation Research and Development Traumatic Brain Injury National Research Center (B9254-C).

\section{Author Disclosure Statement}

No competing financial interests exist.

\section{References}

1. Hoge, C.W., McGurk, D., Thomas, J.L., Cox, A.L., Engel, C.C., and Castro, C.A. (2008). Mild traumatic brain injury in US soldiers returning from Iraq. N. Engl. J. Med. 358, 453-463.

2. Saljo, A., Bao, F., Jingshan, S., Hamberger, A., Hansson, H.-A., and Haglid, K.G. (2002). Exposure to short-lasting impulse noise causes neuronal c-Jun expression and induction of apoptosis in the adult rat brain. J. Neurotrauma 19, 985-991.

3. Goldstein, L.E., Fisher, A.M., Tagge, C.A., Zhang, X.-L., Velisek, L., Sullivan, J.A., Upreti, C., Kracht, J.M., Ericsson, M., Wojnarowicz, M.W., Goletiani, C.J., Maglakelidze, G.M., Case, N., Moncaster, J.A. Minaeva, O., Moir, R.D., Nowinski, C.J., Stern, R.A., Cantu, R.C., Geiling, J., Blusztajn, J.K., Wolozin, B.L., Ikezu, T., Stein, T.D., Budson, A.E., Kowall, N.W., Chargin, D., Sharon, A., Saman, S., Hall, G.F., Moss, W.C., Cleveland, R.O., Tanzi, R.E., Stanton, P.K., and McKee, A.C. (2012). Chronic traumatic encephalopathy in blastexposed military veterans and a blast neurotrauma mouse model. Sci. Transl. Med. 4, ra60.

4. Tompkins, P., Tesiram, Y., Lerner, M., Gonzalez, L.P., Lightfoot, S., Rabb, C.H., and Brackett, D.J. (2013). Brain injury: Neuro-inflammation, cognitive deficit, and magnetic resonance imaging in a model of blast induced traumatic brain injury. J. Neurotrauma 30, 1888-1897.

5. Bazarian, J., Donnelly, K., Peterson, D., Warner, G., Zhu, T., and Zhong, J. (2012) The relation between posttraumatic stress disorder and mild traumatic brain injury acquired during Operations Enduring Freedom and Iraqi Freedom. J. Head Trauma Rehabil. 28, 1-12.

6. Robinson, M.E., Lindemer, E.R., Fonda, J.R., Milberg, W.P., McGlinchey, R.E., and Salat, D.H. (2015) Close-range blast exposure is associated with altered functional connectivity in Veterans independent of concussion symptoms at time of exposure. Hum. Brain Mapp. 36, 911-922.

7. Taber, K., Hurley, R., Haswell, C., Rowland, J., Hurt, S., Lamar, C., and Morey, R. (2015). White matter compromise in veterans exposed to primary blast forces. J. Head Trauma Rehabil. 30, E15-E25.

8. Trotter, B.B., Robinson, M.E., Milberg, W.P., McGlinchey, R.E., and Salat, D.H. (2015. Military blast exposure, ageing, and white matter integrity. Brain 138 2278-2292.

9. Behrens, T.E., and Sporns, O. (2012) Human connectomics. Curr. Opin. Neurobiol. 22, 144-153.

10. Shmuel, A., and Leopold, D.A. (2008) Neuronal correlates of spontaneous fluctuations in fMRI signals in monkey visual cortex: implications for functional connectivity at rest. Hum. Brain Mapp. 29, 751-761.

11. Bright, M.G., and Murphy, K. (2015). Is fMRI "noise"” really noise? Resting state nuisance regressors remove variance with network structure. NeuroImage 114, 158-169.

12. Biswal, B., Yetkin, F., Haughton, V., and Hyde, J. (1995) Functional Connectivity in the motor cortex ofthe resting human brain using echo-planar MRI. Magn. Reson. Med. 34, 537-541.

13. Bandettini, P.A. (2014). Neuronal or hemodynamic? Grappling with the functional MRI signal. Brain Connect. 4, 487-498.

14. Greicius, M.D., Supekar, K., Menon, V., and Dougherty, R.F. (2009). Resting-state functional connectivity reflects structural connectivity in the default mode network. Cereb. Cortex 19, 72-78.

15. Schölvinck, M.L., Maier, A., Frank, Q.Y., Duyn, J.H., and Leopold, D.A. (2010) Neural basis of global resting-state fMRI activity. Proc. Natl. Acad. Sci. 107, 10238-10243.

16. Murphy, K., Harris, A.D., and Wise, R.G. (2011). Robustly measuring vascular reactivity differences with breath-hold: normalising stimulusevoked and resting state BOLD fMRI data. NeuroImage 54, 369-379.

17. D'Esposito, M., Deouell, L.Y., and Gazzaley, A. (2003). Alterations in the BOLD fMRI signal with ageing and disease, a challenge for neuroimaging. Nat. Rev. Neurosci. 4, 863-872.

18. Elder, G.A., and Cristian, A. (2009) Blast-related mild traumatic brain injury: mechanisms of injury and impact on clinical care. Mt. Sinai J. Med. 76, 111-118.

19. Magnuson, J., Leonessa, F., and Ling, G.S. (2012). Neuropathology of explosive blast traumatic brain injury. Curr. Neurol. Neurosci. Rep. 12, 570-579.

20. Lu, J., Ng, K.C., Ling, G., Wu, J., Poon, D.J.F., Kan, E.M., Tan, M.H., Wu, Y.J., Li, P., and Moochhala, S. (2012). Effect of blast exposure on the brain structure and cognition in Macaca fascicularis. J. Neurotrauma 29, 1434-1454.

21. Elder, G.A., Stone, J.R., and Ahlers, S.T. (2014). Effects of low-level blast exposure on the nervous system: is there really a controversy? Front. Neurol. 5, 269.

22. Kamnaksh, A., Kwon, S.Ä., Kovesdi, E., Ahmed, F., Barry, E.S., Grunberg, N.E., Long, J., and Agoston, D. (2012) Neurobehavioral, cellular, and molecular consequences of single and multiple mild blast exposure. Electrophoresis 33, 3680-3692.

23. Huber, B.R., Meabon, J.S., Martin, T.J., Mourad, P.D., Bennett, R., Kraemer, B.C., Cernak, I., Petrie, E.C., Emery, M.J., and Swenson, E.R. (2013). Blast exposure causes early and persistent aberrant phospho-and cleaved-tau expression in a murine model of mild blastinduced traumatic brain injury. J. Alzheimers Dis. 37, 309.

24. Cernak, I., Wang, Z., Jiang, J., Bian, X., and Savic, J. (2001). Cognitive deficits following blast injury-induced neurotrauma: possible involvement of nitric oxide. Brain Inj. 15, 593-612.

25. Readnower, R.D., Chavko, M., Adeeb, S., Conroy, M.D., Pauly, J.R., McCarron, R.M., and Sullivan, P.G. (2010). Increase in blood-brain barrier permeability, oxidative stress, and activated microglia in a rat model of blast-induced traumatic brain injury. J. Neurosci. Res. 88, 3530-3539.

26. Cernak, I., Merkle, A.C., Koliatsos, V.E., Bilik, J.M., Luong, Q.T., Mahota, T.M., Xu, L., Slack, N., Windle, D., and Ahmed, F.A. (2011). The pathobiology of blast injuries and blast-induced neurotrauma as identified using a new experimental model of injury in mice. Neurobiol. Dis. 41, 538-551.

27. Cernak, I. (2010). The importance of systemic response in the pathobiology of blast-induced neurotrauma. Front. Neurol. 1, 151. 
28. Kamnaksh, A., Ahmed, F., Kovesdi, E., Barry, E.S., Grunberg, N.E., Long, J.B., and Agoston, D.V. (2014). Molecular mechanisms of increased cerebral vulnerability after repeated mild blast-induced traumatic brain injury. Transl. Proteom. 3, 22-37.

29. Bir, C., VandeVord, P., Shen, Y., Raza, W., and Haacke, E.M. (2012). Effects of variable blast pressures on blood flow and oxygen saturation in rat brain as evidenced using MRI. Magn. Reson. Imaging 30, 527-534.

30. Shmueli, K., van Gelderen, P., de Zwart, J.A., Horovitz, S.G., Fukunaga, M., Jansma, J.M., and Duyn, J.H. (2007). Low-frequency fluctuations in the cardiac rate as a source of variance in the resting-state fMRI BOLD signal. Neuroimage 38, 306-320.

31. Fox, M.D., Snyder, A.Z., Vincent, J.L., Corbetta, M., Van Essen, D.C., and Raichle, M.E. (2005). The human brain is intrinsically organized into dynamic, anticorrelated functional networks. Proc. Natl. Acad. Sci. U. S. A. 102, 9673-9678.

32. Murphy, K., Birn, R.M., Handwerker, D.A., Jones, T.B., and Bandettini, P.A. (2009. The impact of global signal regression on resting state correlations: are anti-correlated networks introduced? Neuroimage 44, 893-905

33. Gonzalez-Castillo, J., Handwerker, D.A., Robinson, M.E., Hoy, C.W., Buchanan, L.C., Saad, Z.S., and Bandettini, P.A. (2014). The spatial structure of resting state connectivity stability on the scale of minutes. Front. Neurosci. 8, 138

34. Van Dijk, K.R., Hedden, T., Venkataraman, A., Evans, K.C., Lazar, S.W., and Buckner, R.L. (2010). Intrinsic functional connectivity as a tool for human connectomics: theory, properties, and optimization. J. Neurophysiol. 103, 297-321.

35. Handwerker, D.A., Gazzaley, A., Inglis, B.A., and D'Esposito, M. (2007). Reducing vascular variability of fMRI data across aging populations using a breathholding task. Hum. Brain Mapp. 28, 846-859.

36. Hahamy, A., Calhoun, V., Pearlson, G., Harel, M., Stern, N., Attar, F., Malach, R., and Salomon, R. (2014). Save the global: global signal connectivity as a tool for studying clinical populations with functional magnetic resonance imaging. Brain Connect. 4, 395-403.

37. Murphy, K., and Fox, M.D. (2016). Towards a consensus regarding global signal regression for resting state functional connectivity MRI. NeuroImage. [In press.]

38. McGlinchey, R., Milberg, W., Fonda, J., and Fortier, C. (2017). A methodology for assessing deployment trauma and its consequences in OEF/OIF/OND veterans: the TRACTS longitudinal prospective cohort study. Int. J. Methods Psychiatr. Res. [In press.]

39. Fortier, C.B., Amick, M.M., Grande, L., McGlynn, S., Kenna, A., Morra, L., Clark, A., Milberg, W.P., and McGlinchey, R.E. (2014). The Boston Assessment of Traumatic Brain Injury-Lifetime (BAT-L) semistructured interview: evidence of research utility and validity. J. Head Trauma Rehabil. 29, 89-98.

40. Dale, A.M., Fischl, B., and Sereno, M.I. (1999). Cortical surface-based analysis. I. Segmentation and surface reconstruction. Neuroimage 9, 179-194.

41. Fischl, B., Sereno, M.I., and Dale, A.M. (1999) Cortical surface-based analysis. II: Inflation, flattening, and a surface-based coordinate system. Neuroimage 9, 195-207.

42. Fischl, B., Sereno, M.I., Tootell, R.B., and Dale, A.M. (1999) Highresolution intersubject averaging and a coordinate system for the cortical surface. Hum. Brain Mapp. 8, 272-284.

43. Cox, R. (1996). AFNI: software for analysis and visualization of functional magnetic resonance neuroimages. Comput. Biomed. Res. $29,162-173$.
44. Jenkinson, M., Beckmann, C.F., Behrens, T.E., Woolrich, M.W., and Smith, S.M. (2012). FSL. Neuroimage 62, 782-790.

45. Blake, D., Weathers, F., Nagy, L., Kaloupek, D., Gusman, F., Charney, D., and Keane, T. (2006). The development of a clinicianadministered PTSD scale. J. Trauma. Stress 8, 75-90.

46. Fischl, B., Salat, D.H., Busa, E., Albert, M., Dieterich, M., Haselgrove, C., van der Kouwe, A., Killiany, R., Kennedy, D., Klaveness, S., Montillo, A., Makris, N., Rosen, B., and Dale, A.M. (2002). Whole brain segmentation: automated labeling of neuroanatomical structures in the human brain. Neuron 33, 341-355.

47. Abbas, K., Shenk, T., Poole, V., Breedlove, E., Breedlove, K., Leverenz, L., Nauman, E., Talavage, T., and Robinson, M. (2014) Alteration of default mode network in high school football athletes due to repetitive sub-concussive mTBI. Brain Connect. 5, 91-1014.

48. Shively, S.B., Horkayne-Szakaly, I., Jones, R.V., Kelly, J.P., Armstrong, R.C., and Perl, D.P. (2016). Characterisation of interface astroglial scarring in the human brain after blast exposure: a postmortem case series. Lancet Neurol. 15, 944-953.

49. McKee, A.C., and Robinson, M.E. (2014). Military-related traumatic brain injury and neurodegeneration. Alzheimers Dement. 10, S242-S253.

50. Han, K., Mac Donald, C.L., Johnson, A.M., Barnes, Y., Wierzechowski, L., Zonies, D., Oh, J., Flaherty, S., Fang, R., Raichle, M.E., and Brody, D.L. (2014). Disrupted modular organization of restingstate cortical functional connectivity in US military personnel following concussive 'mild' blast-related traumatic brain injury. NeuroImage 84, 76-96.

51. Mac Donald, C.L., Johnson, A.M., Wierzechowski, L., Kassner, E., Stewart, T., Nelson, E.C., Werner, N.J., Zonies, D., Oh, J., Fang, R., and Brody, D.L. (2014). Prospectively assessed clinical outcomes in concussive blast vs nonblast traumatic brain injury among evacuated US military personnel. JAMA Neurol. 71, 994-1002.

52. Mac Donald, C.L., Barber, J., Andre, J., Evans, N., Panks, C., Sun, S., Zalewski, K., Sanders, R.E., and Temkin, N. (2017). 5-Year imaging sequelae of concussive blast injury and relation to early clinical outcome. NeuroImage Clin. 14, 371-378.

53. Hayes, J.P., Miller, D.R., Lafleche, G., Salat, D.H., and Verfaellie, M. (2015). The nature of white matter abnormalities in blast-related mild traumatic brain injury. NeuroImage Clin. 8, 148-156.

54. Davenport, N.D., Lim, K.O., Armstrong, M.T., and Sponheim, S.R. (2012). Diffuse and spatially variable white matter disruptions are associated with blast-related mild traumatic brain injury. Neuroimage 59, 2017-2024.

55. Saad, Z.S., Gotts, S.J., Murphy, K., Chen, G., Jo, H.J., Martin, A., and Cox, R.W. (2012) Trouble at rest: how correlation patterns and group differences become distorted after global signal regression. Brain Connect. 2, 25-32.

Address correspondence to:

Meghan E. Robinson, PhD

Neuroimaging Research for Veterans (NeRVe) Center

VA Boston Healthcare System

150 South Huntington Avenue

Boston, MA 02132

E-mail:dr.meghan.robinson@gmail.com 